\title{
A Controller Design Method under Infrequent, Asynchronous Sensing
}

\author{
Fumin Zhang and Naomi Ehrich Leonard \\ Princeton University, Mechanical and Aerospace Engineering, Princeton, NJ 08544, \\ USA. fzhang, naomi@princeton.edu, \\ WWW home page: http://Www.princeton.edu/ ${ }^{\sim}$ zhang, ${ }^{\sim}$ naomi
}

\begin{abstract}
We use discrete quadratic Lyapunov functions to design controllers for a class of systems where time intervals between state measurements are longer than time intervals between control actions and different components of the state vector are not measured at the same time. The discrete Lyapunov function is a discretization of a continuous Lyapunov function assumed to be known for the idealized system. With this framework, we determine the maximum time interval between measurements of each state variable to guarantee the non-increasing property for the discrete Lyapunov function.
\end{abstract}

\section{Introduction}

We propose a controller design method for systems where the time interval between state measurements is longer than the time interval between control actions. Furthermore, we consider asynchronous sensing which arises naturally from using multiple sensors with different clocks and time scales. We investigate systems where each component in the state vector is updated based on an independent clock, i.e. different state variables are measured at different times. In [11] and [2], asynchronous distributive algorithms and asynchronous multi-agent systems are discussed with a similar definition of asynchronicity. Aysnchronicity is also investigated in [1] and its references. There, however, the asynchronicity relates to non-uniform intervals at which the system is discretized, and state variables are all updated at the same time.

By introducing a formulation of asynchronicity in measurements of different components of the state vector, we establish a procedure that allows us to incorporate sensing asynchronicity in the discrete control Lyapunov function (DCLF) based controller design method. We suppose that for the idealized continuous system when both the control and sensing intervals are infinitely small, a (continuous) controller based on a quadratic control Lyapunov function (CLF) can be found. For the asynchronous system, we use the same CLF as a candidate for the DCLF and show how to change the continuous control law into a discrete law. From this we can estimate an upper bound on the maximum sensing interval for each component in the state. There have been ongoing efforts to generalize Lyapunov stability theory to systems with hybrid nature [4-8]. Our developments allow us to take advantage of the abundant tools available for the continuous design. 


\section{Controller Design}

Consider a continuous system with control on $\mathbb{R}^{n}$ described by $\dot{\mathbf{x}}=f(\mathbf{x})+$ $g(\mathbf{x}) u+F(\mathbf{x}, t)$ where $\mathbf{x}=\left(x_{1}, \ldots, x_{n}\right) \in \mathbb{R}^{n}, u \in \mathbb{R}^{m}$ is the control and $F(\mathbf{x}, t)$ represents the perturbations. Suppose such system has an equilibrium at $\mathbf{x}=0$. It may be possible to find a control Lyapunov function $V(\mathbf{x})$ from which a control law $u$ can be derived to stabilize the equilibrium following [10]. Suppose an estimated perturbation $\hat{F}$ is available. If there exist functions $Y(\mathbf{x})$ and $Z(\mathbf{x})$ such that $Y^{T}(\mathbf{x})=\nabla V^{T} g(\mathbf{x})$ and $Y^{T}(\mathbf{x}) Z(\mathbf{x})=\nabla V^{T} f(\mathbf{x})$ for all $\mathbf{x}$, then $\dot{V}=$ $Y^{T}(\mathbf{x})(\hat{F}(\mathbf{x}, t)+u+Z(\mathbf{x}))+\nabla V^{T} F(\mathbf{x}, t)-Y^{T}(\mathbf{x}) \hat{F}(\mathbf{x}, t)$. The design of $u$ is $u=-Z(\mathbf{x})-K Y(\mathbf{x})-\hat{F}(\mathbf{x}, t)$.

We assume that the control interval $\Delta t$, the time between two control actions, is fixed. We also assume each sensing interval $\Delta T_{i}$, the time between measurements of $x_{i}$, is fixed and $\Delta T_{i}=P_{i} \Delta t$ where $P_{i}>1$ is an integer for $i=1, \ldots, n$. A simple recursive Euler predictor can be employed to estimate the states from measurements: $\mathbf{x}_{k+1}^{-}=\mathbf{x}_{k}^{+}+f\left(\mathbf{x}_{k}^{+}\right) \Delta t+g\left(\mathbf{x}_{k}^{+}\right) u_{k} \Delta t+\hat{F}\left(\mathbf{x}_{k}^{+}, t_{k}\right) \Delta t$. The symbol $\mathbf{x}_{k}^{+}$represents the updated state at step $k$ that has incorporated available sensor information. The symbol $\mathbf{x}_{k+1}^{-}$represents the predicted state at step $k+1$. If no measurements are taken at step $k$, then we let $\mathbf{x}_{k}^{+}=\mathbf{x}_{k}^{-}$. Note that more sophisticated predictors can be applied [9].

Suppose we use a discretized control law: $u_{k}=-Z\left(\mathbf{x}_{k}^{+}\right)-K Y\left(\mathbf{x}_{k}^{+}\right)-\hat{F}\left(\mathbf{x}_{k}^{+}, t_{k}\right)$. Suppose further that the Lyapunov function $V$ is quadratic. Then $V_{k+1}^{-}-V_{k}^{+}=$ $\nabla\left(V_{k}^{+}\right)^{T}\left(\mathbf{x}_{k+1}^{-}-\mathbf{x}_{k}^{+}\right)+\frac{1}{2}\left(\mathbf{x}_{k+1}^{-}-\mathbf{x}_{k}^{+}\right)^{T} H_{k}^{+}\left(\mathbf{x}_{k+1}^{-}-\mathbf{x}_{k}^{+}\right)$where $\nabla V_{k}^{+}$and $H_{k}^{+}$ represents the gradient and Hessian of function $V$ at $\mathbf{x}_{k}^{+}$.

Suppose the estimate of the perturbation satisfies $Y^{T} \hat{F}-\nabla V^{T} F=0$ when measurements are taken. Under the proposed control we have

$$
V_{k+1}^{-}-V_{k}^{+}=-K Y_{k}^{T} Y_{k} \Delta t+\left\|f_{k}+F_{k}-g_{k}\left(Z_{k}+\hat{F}_{k}+K Y_{k}\right)\right\|_{H_{k}^{+}}^{2} \Delta t^{2}
$$

where we let $F_{k}=F\left(\mathbf{x}_{k}^{+}, t_{k}\right)$ and $g_{k}, Y_{k}, Z_{k}$ and $f_{k}$ are defined similarly. Matrix $H_{k}^{+}$defines a pseudo inner product $\langle\cdot, \cdot\rangle_{H_{k}^{+}}$and its induced norm $\|\cdot\|_{H_{k}^{+}}$.

From (1) we can determine the value of $K$ that achieves the largest decrease in $V$ as $K_{k}=\left(2\left\langle A_{k}, g_{k} Y_{k}\right\rangle_{H_{k}^{+}} \Delta t+Y_{k}^{T} Y_{k}\right) /\left(2\left\|g_{k} Y_{k}\right\|_{H_{k}^{+}}^{2} \Delta t\right)$ where $A_{k}=f_{k}+$ $F_{k}-g_{k}\left(Z_{k}+\hat{F}_{k}\right)$. The maximum decrease in $V$ is

$$
\left(V_{k+1}^{-}-V_{k}^{+}\right)^{*}=\left\|A_{k}\right\|_{H_{k}^{+}}^{2} \Delta t^{2}-\frac{\left(2\left\langle A_{k}, g_{k} Y_{k}\right\rangle_{H_{k}^{+}} \Delta t+Y_{k}^{T} Y_{k}\right)^{2}}{4\left\|g_{k} Y_{k}\right\|_{H_{k}^{+}}^{2}} .
$$

\section{Asynchronous Sensing}

If there exist rational numbers $a_{i l}$ such that $\Delta T_{i}=a_{i l} \Delta T_{l}$ for all $i, l=1, \ldots, n$, we say the asynchronous sensing is resonant. By assumptions made in Sec. 2 we develop results for the resonant case. 
Let $j_{m}$ and $j_{m+1}$ be two time indices. We use the symbol $\Gamma_{i}\left(j_{m}, j_{m+1}\right)$ to represent the set of time indices when measurements for $x_{i}$ are available within the interval $\left[j_{m}, j_{m+1}\right)$. In the resonant case, we can always find an (infinite) sequence $\left\{j_{m}\right\}$ such that: (S1) The interval $j_{m+1}-j_{m}$ is constant for all $m$. (S2) $\Gamma_{i}\left(j_{m}, j_{m+1}\right)$ is not empty for all $i$. (S3) There exists $i$ such that a measurement for $x_{i}$ is available at each $j_{m}$.

We often write $\Gamma_{i}\left(j_{m}, j_{m+1}\right)$ simply as $\Gamma_{i}$. Let $N_{i}$ be the total number of elements in $\Gamma_{i}$ and let $N=\sum_{i} N_{i}$. Since $N$ is finite, we can construct an $N$ dimensional vector $\mathbf{v}$ that contains all members of $\Gamma_{i}$ for all $i$. We call $\mathbf{v}$ the asynchronous index vector. Note that $N$ is constant for all $\left[j_{m}, j_{m+1}\right)$.

Consider the DCLF $V(\mathbf{x})$ where $\mathbf{x} \in \mathbb{R}^{n}$. Let $p$ assume all possible values for $p \in \Gamma_{i}$ and for $i=1,2, \ldots, n$. We use $V_{p}^{-}$and $V_{p}^{+}$to denote the function value before and after the update. Let $\mathbf{x}_{p}^{-}, \mathbf{x}_{p}^{+}, \nabla V_{p}^{-}$and $H_{p}^{-}$be the state vector before measurement, the state vector after measurement, the gradient vector and the Hessian at the time instant indexed by $p$.

Definition 1. Consider the interval $\left[j_{m}, j_{m+1}\right)$. On this interval, we define the asynchronous state vector before update as the $N$ dimensional vector $\hat{\mathbf{x}}^{\mathrm{a}}$ whose $r$-th component satisfies $\left(\hat{\mathbf{x}}^{\mathrm{a}}\right)_{r}=\left(\mathbf{x}_{v_{r}}^{-}\right)_{i}$ where $v_{r}$ is the $r$-th component of the asynchronous index vector $\mathbf{v}$ and $v_{r} \in \Gamma_{i}$. We define the asynchronous state vector after update as the $N$ dimensional vector $\mathbf{x}^{\mathrm{a}}$ whose $r$-th component satisfies $\left(\mathbf{x}^{\mathrm{a}}\right)_{r}=\left(\mathbf{x}_{v_{r}}^{+}\right)_{i}$. We define the asynchronous gradient as the $N$ dimensional vector $\nabla^{\mathrm{a}} V$ whose $r$-th component satisfies $\left(\nabla^{\mathrm{a}} V\right)_{r}=\left(\nabla V_{v_{r}}^{-}\right)_{i}$. We define the asynchronous Hessian as the $N \times N$ matrix $H^{\text {a }}$ whose elements satisfy, for $r, l=1,2, \ldots, N,\left(H^{\mathrm{a}}\right)_{r, l}=\left(H_{v_{r}}^{-}\right)_{i_{1}, i_{1}}$ if $r=l,\left(H^{\mathrm{a}}\right)_{r, l}=\left(H_{v_{r}}^{-}\right)_{i_{1}, i_{2}}$ if $v_{r}=v_{l}$ but $i_{1} \neq i_{2}$, and $\left(H^{\mathrm{a}}\right)_{r, l}=0$ for other cases, where indices $i_{1}$ and $i_{2}$ are such that $v_{r} \in \Gamma_{i_{1}}$ and $v_{l} \in \Gamma_{i_{2}}$.

As a convention, we let $V_{p}^{-}=V_{p}^{+}$if $p \notin \Gamma_{i}$ for any $i$. We compare the value of the candidate function $V$ at two time instants $j_{m}$ and $j_{m+1}$ when the same part of the states have been updated. The difference between the function values $V_{j_{m}}^{-}$and $V_{j_{m+1}}^{-}$can be written as $V_{j_{m+1}}^{-}-V_{j_{m}}^{-}=\sum_{p=j_{m}}^{j_{m+1}-1}\left(V_{p}^{+}-V_{p}^{-}\right)+$ $\sum_{p=j_{m}}^{j_{m+1}-1}\left(V_{p+1}^{-}-V_{p}^{+}\right)$. Using the discretized controller $u_{k}$ with adaptive gain $K_{k}$ from Sec. 2, for $V_{j_{m+1}}^{-}-V_{j_{m}}^{-} \leq 0$ to hold, we need $\sum_{p=j_{m}}^{j_{m+1}-1}\left(V_{p}^{+}-V_{p}^{-}\right) \leq$ $\sum_{p=j_{m}}^{j_{m+1}-1}\left(V_{p}^{+}-V_{p+1}^{-}\right)^{*}$. We introduce the notion of an asynchronously positive invariant set. This notion is closely related to Poisson stability c.f. [3].

Definition 2. Consider an infinite sequence $\left\{j_{m}\right\}$ that satisfies assumptions (S1-S3). We say a set $M \subset \mathbb{R}^{N}$ is asynchronously positive invariant for $\left\{j_{m}\right\}$ if for $\mathbf{x}^{a}\left(j_{0}, j_{1}\right) \in M$, we have $\mathbf{x}^{\mathrm{a}}\left(j_{m}, j_{m+1}\right) \in M$ for all $m \geq 0$.

We let the set $E_{1} \subset \mathbb{R}^{N}$ be the set of all asynchronous states $\mathrm{x}^{\mathrm{a}}$ where $V_{j_{m+1}}^{-}-V_{j_{m}}^{-} \leq 0$ is satisfied for the sequence $\left\{j_{m}\right\}$. Let the set $M_{1}$ be the set of $\mathbf{x}^{\mathrm{a}}$ where $V\left(\Phi_{j_{m+1}}^{-}\left(\mathbf{x}^{\mathrm{a}}\left(j_{m}, j_{m+1}\right)\right)\right) \leq c_{1}$ for some $c_{1} \geq 0$ where $\Phi_{j_{m+1}}^{-}$is the state transition map from $\mathbf{x}^{\mathrm{a}}\left(j_{m}, j_{m+1}\right)$ to $\mathbf{x}_{j_{m+1}}^{-}$.

Proposition 1. If $M_{1} \subset E_{1}$, then $M_{1}$ is asynchronously positive invariant. 
Let $\mathbf{e}^{\mathrm{a}}=\mathbf{x}^{\mathrm{a}}-\hat{\mathbf{x}}^{\mathrm{a}}$. This $\mathbf{e}^{\mathrm{a}}$ contains all the corrections to the states when measurements are available. To estimate the upper bound for sensing intervals, some assumptions are needed regarding how the prediction error depends on time. If $x_{i}^{+}(t)=x_{i}^{-}(t)$, we assume that $\left|x_{i}^{+}(t+\tau)-x_{i}^{-}(t+\tau)\right| \leq L \tau^{q}$ for $i=1,2, \ldots, n$ where $L>0, q \geq 1$ and for all $t, \tau \geq 0$. Under this assumption, the asynchronous error satisfies $\left\|\mathbf{e}^{\mathrm{a}}\right\| \leq L \Delta T_{i}^{q}\left(\sum_{l=1}^{n}\left(N_{l} a_{l i}\right)^{2}\right)^{\frac{1}{2}}$ for any given $i$.

Proposition 2. $\sum_{p=j_{m}}^{j_{m+1}-1}\left(V_{p}^{+}-V_{p}^{-}\right)=\left(\nabla^{\mathrm{a}} V\right)^{T} \mathbf{e}^{\mathrm{a}}+\frac{1}{2}\left(\mathbf{e}^{\mathrm{a}}\right)^{T} H^{\mathrm{a}} \mathbf{e}^{\mathrm{a}}$.

We now know $\left|\sum_{p=j_{m}}^{j_{m+1}-1}\left(V_{p}^{+}-V_{p}^{-}\right)\right| \leq\left\|\nabla V^{\mathrm{a}}\right\|\left\|\mathbf{e}^{\mathrm{a}}\right\|+\frac{1}{2}\left\|H^{\mathrm{a}}\right\|\left\|\mathbf{e}^{\mathrm{a}}\right\|^{2}$. Then a sufficient condition for $V_{j_{m+1}}^{-}-V_{j_{m}}^{-} \leq 0$ is $\left\|\nabla V^{\mathrm{a}}\right\|\left\|\mathbf{e}^{\mathrm{a}}\right\|+\frac{1}{2}\left\|H^{\mathrm{a}}\right\|\left\|\mathbf{e}^{\mathrm{a}}\right\|^{2} \leq$ $\Delta V^{*}$ where $\Delta V^{*}=\sum_{p=j_{m}}^{j_{m+1}-1}\left(V_{p}^{+}-V_{p+1}^{-}\right)^{*}$. If this condition is satisfied for all $\mathbf{x}^{a} \in M_{1}$, then $M_{1} \subset E_{1}$ and $M_{1}$ is asynchronously positive invariant by Prop. 1 . We can estimate the maximum sensing interval $\Delta T_{i}$ for $M_{1}$ to be asynchronously positive invariant as

$$
\Delta T_{i}^{*}=\inf _{\mathbf{x}^{\mathrm{a}} \in M_{1}}\left(\frac{-\left\|\nabla V^{\mathrm{a}}\right\|+\left(\left\|\nabla V^{\mathrm{a}}\right\|^{2}+2\left\|H^{\mathrm{a}}\right\| \Delta V^{*}\right)^{\frac{1}{2}}}{L\left(\sum_{l=1}^{n}\left(N_{l} a_{l i}\right)^{2}\right)^{\frac{1}{2}}\left\|H^{\mathrm{a}}\right\|}\right)^{\frac{1}{q}} .
$$

\section{References}

1. A. Balluchi, P. Murrieri, and A. L. Sangiovanni-Vincentelli. Controller synthesis on non-uniform and uncertain discrete-time domains. In M. Morari and L. Thiele, editors, HSCC 2005, LNCS 3414:118-133. Springer-Verlag, 2005.

2. M. Cao, A. S. Morse, and B.D.O. Anderson. Agreeing asynchronously in continuous time, IEEE Trans. Automat. Cont., submitted 2006.

3. P. E. Crouch. Spacecraft attitude control and stabilization: applications of geometric control theory to rigid body models. IEEE Trans. Automat. Cont., 29(4):321331,1984 .

4. J.W. Grizzle and J-M. Kang. Discrete-time control design with positive semidefinite Lyapunov functions. Syst. \& Cont. Letters, 40(3):329-342, 1984.

5. T. Hu and Z. Lin. Absolute stability analysis of discrete-time systems with composite quadratic Lyapunov functions. IEEE Trans. Automat. Cont., 50(6):781-797, 2005.

6. C.J.B. Macnab and G.M.T. D'Eleuterio. Discrete-time Lyapunov design for neuroadaptive control of elastic-joint robots. Int. J. Robot. Res., 19(5):511-525, 2000.

7. K. M. Passino, A. N. Michel, and P. J. Antsaklis. Lyapunov stability of a class of discrete event systems. IEEE Trans. Automat. Cont., 39(2):269-279, 1994.

8. M. S. Branicky. Multiple Lyapunov functions and other analysis tools for switched and hybrid systems. IEEE Trans. Automat. Cont., 43:475-482, 1998.

9. B. Sinopoli, L. Schenato, M. Franceschetti, K. Poolla, M. Jordan and S. Sastry. Kalman filtering with intermittent observations IEEE Trans. Automat. Cont., 49(9):1453-1464, 2004.

10. E. D. Sontag. Mathematical Control Theory: Deterministic Finite Dimensional Systems 2nd ed., Springer, 1998.

11. J. N. Tsitsiklis and D. P. Bertsekas. Distributed asynchronous routing in data communication networks. IEEE Trans. Automat. Cont., 31:325-332, 1986. 